\title{
Excited bands in odd-mass rare-earth nuclei
}

\author{
Carlos E. Vargas \\ Grand Accélérateur National d'Ions Lourds, BP 502\%, F-14076 Caen Cedex 5, France \\ Jorge G. Hirsch \\ Instituto de Ciencias Nucleares, Universidad Nacional Autónoma de México, \\ Apartado Postal 70-543 México 04510 DF, México \\ Jerry P. Draayer用 \\ Department of Physics and Astronomy, Louisiana State University, Baton Rouge, Louisiana 70803, U.S.A.
}

(Dated: November 5, 2018)

\begin{abstract}
Normal parity bands are studied in ${ }^{157} \mathrm{Gd},{ }^{163} \mathrm{Dy}$ and ${ }^{169} \mathrm{Tm}$ using the pseudo SU(3) shell model. Energies and B(E2) transition strengths of states belonging to six low-lying rotational bands with the same parity in each nuclei are presented. The pseudo SU(3) basis includes states with pseudo-spin 0 and 1 , and $\frac{1}{2}$ and $\frac{3}{2}$, for even and odd number of nucleons, respectively. States with pseudo-spin 1 and $\frac{3}{2}$ must be included for a proper description of some excited bands.

PACS numbers: 21.60.Cs, 21.60.Fw, 23.20.Lv, 27.70.+q
\end{abstract}

\section{INTRODUCTION}

The nuclear shell model [1] is the cornerstone in the microscopic study of nuclear properties. In the last two decades it has been possible to perform calculations for valence nucleons in the full $s d-$ [2] and $f p-$ [3] shells, providing a detailed description of energy levels, and electromagnetic and weak transitions. Nevertheless, a shell model description of heavy nuclei requires further assumptions that include a systematic and proper truncation of the model space [4].

In light deformed nuclei the dominance of quadrupolequadrupole interaction led to the introduction of the SU(3) shell model [5]. However, the strong spin-orbit interaction renders the $\mathrm{SU}(3)$ truncation scheme useless in heavier nuclei, while at the same time pseudo-spin emerges as a good symmetry [6], and with it the pseudo$\mathrm{SU}(3)$ model [7].

Pseudo-spin symmetry refers to the experimental fact that single-particle orbitals with $j=l-\frac{1}{2}$ and $j=$ $(l-2)+\frac{1}{2}$ in the shell $\eta$ lie very close in energy, and can therefore be labeled as pseudo-spin doublets with quantum numbers $\tilde{\jmath}=j, \tilde{\eta}=\eta-1$, and $\tilde{l}=l-1$. The origin of this symmetry has been traced back to the relativistic mean field equations [8].

During the last decade the pseudo-SU(3) shell model has significantly evolved, becoming a powerful microscopic theory for the description of the low energy rotational bands in even-even heavy deformed nuclei, and of normal parity bands in heavy deformed odd-A nuclei. The first applications considered pseudo-SU(3) a dynam-

\footnotetext{
*Electronic address: vargas@ganil.fr

${ }^{\dagger}$ Electronic address: hirsch@nuclecu.unam.mx

‡Electronic address: draayer@lsu.edu
}

ical symmetry, using only one irreducible representation (irrep) of $\mathrm{SU}(3)$ to describe the yrast band up to the backbending regime [7]. After many improvements in the rotor Hamiltonian, a technical breakthrough came with the development of a computer code able to calculate reduced matrix elements of physical operators between different SU(3) irreps [9]. From then on it was possible to use a realistic Hamiltonian which, besides the quadrupole-quadrupole interaction and rotor terms, includes the breaking symmetry single particle energies and pairing interactions.

In this way a fully microscopic description of lowenergy bands in even-even and odd-A heavy deformed nuclei emerged. The model have been using as building blocks the pseudo-SU(3) proton and neutron states having pseudo-spin 0 and $\frac{1}{2}$, which describe the even- and odd-A systems, respectively. The many-particle states are built as linear combinations of pseudo-SU(3) coupled states with well-defined particle number and total angular momentum. Many rotational bands and $\mathrm{B}(\mathrm{E} 2)$ electromagnetic transition strengths have been described in the even-even rare earth isotopes ${ }^{156,158,160} \mathrm{Gd}$, ${ }^{160,162,164}$ Dy and ${ }^{164,166,168} \operatorname{Er}[10,11,12$ and in the oddmass ${ }^{159,161} \mathrm{~Tb},{ }^{159,163} \mathrm{Dy},{ }^{159} \mathrm{Eu},{ }^{161} \mathrm{Tm}$, and ${ }^{165,167} \mathrm{Er}$ nuclei 13, 14, 15, 16.

Widening the landscape of applications of the pseudo $\mathrm{SU}(3)$ model, in the present work proton and neutron states having pseudo spin 1 and $\frac{3}{2}$, for even and odd number of particles, respectively, are included in the Hilbert space. The final many-particle states in odd-mass nuclei have total spin $\frac{1}{2}, \frac{3}{2}$ or $\frac{5}{2}$. In this enriched space, six or seven normal parity low-lying rotational bands in ${ }^{157} \mathrm{Gd}$, ${ }^{163} \mathrm{Dy}$ and ${ }^{169} \mathrm{Tm}$ are successfully described. Many of them have important pseudo-spin 1 and $\frac{3}{2}$ components, evidencing their relevance in the present study. Intra and inter-band $\mathrm{B}(\mathrm{E} 2)$ transition strengths are also discussed. This article complements the research of the scis- 
sors mode, M1 transition strengths in the range between 2 and $4 \mathrm{MeV}$, performed in the same nuclei [17].

In Section II a brief description of the pseudo SU(3) classification scheme is presented. The schematic Hamiltonian and its parametrization is discussed in Section III. The results for low-lying rotational bands in ${ }^{157} \mathrm{Gd},{ }^{163} \mathrm{Dy}$ and ${ }^{169} \mathrm{Tm}$ are presented in Section IV, where their wave functions are also discussed. The B(E2) intra- and interband transition strengths are presented in Section V. The summary and conclusions are given in Section VI

\section{THE PSEUDO SU(3) BASIS}

The starting point in any application of the pseudo $\mathrm{SU}(3)$ model is the building of the many-body basis. The proton and neutron valence Nilsson single particle levels are filled from below for a fixed deformation, allowing the determination of the occupancies in normal and unique parity orbitals [15]. As it has been the case for all pseudo $\mathrm{SU}(3)$ studies up to now, nucleons in abnormal parity orbital are frozen, being the dynamics described using only nucleons in normal parity states. This choice is further reflected through the use of effective charges to describe quadrupole electromagnetic transitions which are larger than those usually employed in shell-model calculations. While it has been shown that this is a reasonable and useful approach, it is nonetheless a strong assumption and the most important limitation of the present model. A more sophisticated treatment of the problem, with nucleons in intruder orbitals described in the same footing using $\mathrm{SU}(3)$ irreps is under development [18, 19].

In Table 1 are presented the occupation numbers assigned to each nuclei.

\begin{tabular}{c|c|cccc} 
Nuclei & $\beta$ & $n_{\pi}^{N}$ & $n_{\nu}^{N}$ & $n_{\pi}^{A}$ & $n_{\nu}^{A}$ \\
\hline${ }^{157} \mathrm{Gd}$ & 0.250 & 8 & 7 & 6 & 4 \\
${ }^{163} \mathrm{Dy}$ & 0.258 & 10 & 9 & 6 & 6 \\
${ }^{169} \mathrm{Tm}$ & 0.267 & 11 & 12 & 8 & 6
\end{tabular}

TABLE I: Deformation and occupation numbers.

The many-particle states of $n_{\alpha}$ active nucleons in a given normal parity shell $\eta_{\alpha}, \alpha=\nu$ or $\pi$, can be classified by the following chains of groups:

$$
\begin{aligned}
& \left\{1^{n_{\alpha}^{N}}\right\} \quad\left\{\tilde{f}_{\alpha}\right\} \quad\left\{f_{\alpha}\right\} \gamma_{\alpha}\left(\lambda_{\alpha}, \mu_{\alpha}\right) \quad \tilde{S}_{\alpha} \kappa_{\alpha} \\
& U\left(\Omega_{\alpha}^{N}\right) \supset U\left(\Omega_{\alpha}^{N} / 2\right) \times U(2) \supset S U(3) \times S U(2) \supset \\
& \tilde{L}_{\alpha} \quad J_{\alpha}^{N} \\
& S O(3) \times S U(2) \supset S U_{J}(2),
\end{aligned}
$$

where above each group the quantum numbers that characterize its irreps are given, and $\gamma_{\alpha}$ and $\kappa_{\alpha}$ are multiplicity labels of the indicated reductions.

Any state $\left|J_{i} M\right\rangle$, where $J$ is the total angular momentum, $M$ its projection and $i$ an integer index which enumerates the states with the same $J, M$ starting from the one with the lowest energy, is built as a linear combination

$$
\left|J_{i} M\right\rangle=\sum_{\beta} C_{\beta}^{J_{i}}|\beta J M\rangle
$$

of the strong coupled proton-neutron states

$$
\begin{array}{r}
|\beta J M\rangle \equiv \\
\left|\left\{\tilde{f}_{\pi}\right\}\left(\lambda_{\pi} \mu_{\pi}\right) \tilde{S}_{\pi},\left\{\tilde{f}_{\nu}\right\}\left(\lambda_{\nu} \mu_{\nu}\right) \tilde{S}_{\nu} ; \rho(\lambda \mu) \kappa L, \tilde{S} J M\right\rangle= \\
\sum_{M_{L} M_{S}}\left(L M_{L}, \tilde{S} M_{S} \mid J M\right) \sum_{M_{S \pi} M_{S \nu}}\left(\tilde{S}_{\pi} M_{S \pi}, \tilde{S}_{\nu} M_{S \nu} \mid \tilde{S} M_{S}\right) \\
\sum_{k_{\pi} L_{\pi} M_{\pi}}\left\langle\left(\lambda_{\pi} \mu_{\pi}\right) \kappa_{\pi} L_{\pi} M_{\pi} ;\left(\lambda_{\nu} \mu_{\nu}\right) \kappa_{\nu} L_{\nu} M_{\nu} \mid(\lambda \mu) \kappa L M\right\rangle_{\rho} \\
\kappa_{\nu} L_{\nu} M_{\nu} \\
\left.\left.\qquad \tilde{f}_{\pi}\right\}\left(\lambda_{\pi} \mu_{\pi}\right) \kappa_{\pi} L_{\pi} M_{\pi}, \tilde{S}_{\pi} M_{S \pi}\right\rangle \\
\left|\left\{\tilde{f}_{\nu}\right\}\left(\lambda_{\nu} \mu_{\nu}\right) \kappa_{\nu} L_{\nu} M_{\nu}, \tilde{S}_{\nu} M_{S \nu}\right\rangle
\end{array} .
$$

In the above expression $\langle-;-\mid-\rangle$ and $(-,-\mid-)$ are the $\mathrm{SU}(3)$ and $\mathrm{SU}(2)$ Clebsch Gordan coefficients, respectively.

In all previous applications of the pseudo SU(3) model, only those states with the highest spatial symmetry $\tilde{S}_{\pi, \nu}$ $=0$ and $\frac{1}{2}$ have been included. In the present study states with $\tilde{S}_{\pi, \nu}=1$ and $\frac{3}{2}$ are also taken into account, allowing for coupled proton-neutron states with total pseudospin $\tilde{S}=\frac{1}{2}, \frac{3}{2}$ or $\frac{5}{2}$. The pseudo-spin symmetry is close enough to an exact symmetry in atomic nuclei, supporting a strong truncation of the Hilbert space. However, pseudo spin-orbit partners are not exactly degenerate, introducing a small degree of pseudo-spin mixing in the nuclear wave function.

The quadrupole-quadrupole interaction can be expressed in terms of the second order SU(3) Casimir operator $C_{2}$,

$$
\hat{Q} \cdot \hat{Q}=4 C_{2}-3 \hat{L}(\hat{L}+1) .
$$

The eigenvalue of $C_{2}$ for a given of $\mathrm{SU}(3)$ irrep $(\lambda, \mu)$ is given by

$$
\left\langle C_{2}\right\rangle=\lambda^{2}+\mu^{2}+\lambda \mu+3 \lambda+3 \mu .
$$

The larger the expectation value of $C_{2}$, the greater the binding of that $\mathrm{SU}(3)$ irrep by a pure attractive $Q \cdot Q$ interaction. The pseudo $\mathrm{SU}(3)$ basis is built selecting those proton and neutron irreps with the largest $\left\langle C_{2}\right\rangle$ and pseudo-spin 0 and 1 , or $\frac{1}{2}$ and $\frac{3}{2}$, for even and odd number of particles, respectively. The proton and neutron irreps are coupled to a total pseudo $\mathrm{SU}(3)(\lambda, \mu)$ irrep and to a total pseudo-spin $\tilde{S}=\frac{1}{2}, \frac{3}{2}$ and $\frac{5}{2}$. The basis states employed in the present work for each of the three nuclei studied are presented in Tables II] [II] and [V.

\section{THE PSEUDO SU(3) HAMILTONIAN}

The Hamiltonian contains spherical Nilsson singleparticle terms for protons $\left(H_{s p, \pi}\right)$ and neutrons $\left(H_{s p, \nu}\right)$, 


\begin{tabular}{ll|lll}
$\left(\lambda_{\pi}, \mu_{\pi}\right) S_{\pi}$ & $\left(\lambda_{\nu}, \mu_{\nu}\right) S_{\nu}$ & \multicolumn{3}{|c}{ total $(\lambda, \mu)$} \\
\hline$(10,4) 0$ & $(15,5) \frac{1}{2}$ & $(25,9) \frac{1}{2}$ & $(27,5) \frac{1}{2}$ & $(26,7) \frac{1}{2}$ \\
$(10,4) 0$ & $(18,2) \frac{1}{2}$ & $(27,5) \frac{1}{2}$ & $(26,7) \frac{1}{2}$ & $(29,4) \frac{1}{2}$ \\
& & $(28,6) \frac{1}{2}$ & $(30,2) \frac{1}{2}$ & \\
$(10,4) 0$ & $(16,3) \frac{1}{2}, \frac{3}{2}$ & $(27,5) \frac{1}{2}, \frac{3}{2}$ & $(26,7) \frac{1}{2}, \frac{3}{2}$ & \\
$(10,4) 0$ & $(17,1) \frac{1}{2}, \frac{3}{2}$ & $(27,5) \frac{1}{2}, \frac{3}{2}$ & & \\
$(11,2) 1$ & $(18,2) \frac{1}{2}$ & $(27,5) \frac{1}{2}, \frac{3}{2}$ & $(29,4) \frac{1}{2}, \frac{3}{2}$ & \\
& & $(31,0) \frac{1}{2}, \frac{3}{2}$ & $(30,2) \frac{1}{2}, \frac{3}{2}$ & \\
$(11,2) 1$ & $(15,5) \frac{1}{2}$ & $(27,5) \frac{1}{2}, \frac{3}{2}$ & $(26,7) \frac{1}{2}, \frac{3}{2}$ \\
$(11,2) 1$ & $(16,3) \frac{1}{2}, \frac{3}{2}$ & $(27,5) \frac{1}{2}, \frac{3}{2}, \frac{5}{2}$ & \\
$(12,0) 0$ & $(15,5) \frac{1}{2}$ & $(27,5) \frac{1}{2}$ & \\
$(12,0) 0$ & $(18,2) \frac{1}{2}$ & $(30,2) \frac{1}{2}$ & \\
$(8,5) 0,1$ & $(18,2) \frac{1}{2}$ & $(26,7) \frac{1}{2}, \frac{3}{2}$ & $(27,5) \frac{1}{2}, \frac{3}{2}$ \\
$(9,3) 0,1$ & $(18,2) \frac{1}{2}$ & $(27,5) \frac{1}{2}, \frac{3}{2}$ &
\end{tabular}

TABLE II: The pseudo SU(3) irreps used in the description of ${ }^{157} \mathrm{Gd}$.

\begin{tabular}{ll|lll}
$\left(\lambda_{\pi}, \mu_{\pi}\right) S_{\pi}$ & $\left(\lambda_{\nu}, \mu_{\nu}\right) S_{\nu}$ & \multicolumn{3}{|c}{ total $(\lambda, \mu)$} \\
\hline$(10,4) 0$ & $(16,7) \frac{1}{2}$ & $(26,11) \frac{1}{2}$ & & \\
$(7,7) 0,1$ & $(19,4) \frac{1}{2}$ & $(26,11) \frac{1}{2}, \frac{3}{2}$ & & \\
$(10,4) 0$ & $(19,4) \frac{1}{2}$ & $(29,8) \frac{1}{2}$ & $(31,4) \frac{1}{2}$ & $(30,6) \frac{1}{2}$ \\
& & $(32,2) \frac{1}{2}$ & & \\
$(10,4) 0$ & $(20,2) \frac{1}{2}$ & $(31,4) \frac{1}{2}$ & $(30,6) \frac{1}{2}$ & $(32,2) \frac{1}{2}$ \\
$(10,4) 0$ & $(21,0) \frac{3}{2}$ & $(31,4) \frac{3}{2}$ & & \\
$(11,2) 1$ & $(19,4) \frac{1}{2}$ & $(31,4) \frac{1}{2}, \frac{3}{2}$ & $(30,6) \frac{1}{2}, \frac{3}{2}$ & $(32,2) \frac{1}{2}, \frac{3}{2}$ \\
$(11,2) 1$ & $(20,2) \frac{1}{2}$ & $(31,4) \frac{1}{2}, \frac{3}{2}$ & $(32,2) \frac{1}{2}, \frac{3}{2}$ \\
$(12,0) 0$ & $(19,4) \frac{1}{2}$ & $(31,4) \frac{1}{2}$ & \\
$(11,2) 1$ & $(21,0) \frac{3}{2}$ & $(32,2) \frac{1}{2}, \frac{3}{2}, \frac{5}{2}$ & \\
$(12,0) 0$ & $(20,2) \frac{1}{2}$ & $(32,2) \frac{1}{2}$ &
\end{tabular}

TABLE III: The pseudo SU(3) irreps used in the description of ${ }^{163}$ Dy.

the quadrupole-quadrupole $(\tilde{Q} \cdot \tilde{Q})$ and pairing interactions $\left(H_{p a i r, \pi}\right.$ and $\left.H_{p a i r, \nu}\right)$, as well as three 'rotor-like' terms which are diagonal in the $\mathrm{SU}(3)$ basis.

$$
\begin{aligned}
H= & \sum_{\alpha=\pi, \nu}\left\{H_{s p, \alpha}-G_{\alpha} H_{\text {pair }, \alpha}\right\}-\frac{1}{2} \chi \tilde{Q} \cdot \tilde{Q} \\
& +a K_{J}^{2}+b J^{2}+A_{\text {sym }} \hat{C}_{2} .
\end{aligned}
$$

This Hamiltonian can be separated into two parts: the first row includes Nilsson single-particle energies and the pairing and quadrupole-quadrupole interactions $(\tilde{Q}$ is the quadrupole operator in the pseudo $\mathrm{SU}(3)$ space, see below). They are the basic components of any realistic Hamiltonian and have been widely studied in the nuclear physics literature, allowing their respective strengths to be fixed by systematics [20, 21]. The $\mathrm{SU}(3)$ mixing is due to the single-particle and pairing terms. The second row

\begin{tabular}{|c|c|c|c|}
\hline$\left(\lambda_{\pi}, \mu_{\pi}\right) S$ & $\left(\lambda_{\nu}, \mu_{\nu}\right) S_{\nu}$ & & otal $(\lambda, \mu)$ \\
\hline$(7,7) \frac{1}{2}$ & $(24,0) 0$ & $(31,7) \frac{1}{2}$ & \\
\hline$(11,2) \frac{1}{2}$ & $(20,5) 0,1$ & $(31,7) \frac{1}{2}, \frac{3}{2}$ & $(33,3) \frac{1}{2}, \frac{3}{2} \quad(32,5) \frac{1}{2}, \frac{3}{2}$ \\
\hline$(7,7) \frac{1}{2}$ & $(16,10) 0$ & $(23,17) \frac{1}{2}$ & \\
\hline$(11,2) \frac{1}{2}$ & $(24,0) 0$ & $(33,3) \frac{1}{2}$ & $(34,1) \frac{1}{2} \quad(35,2) \frac{1}{2}$ \\
\hline$(11,2) \frac{1}{2}$ & $(21,3) 0,1$ & $(33,3) \frac{1}{2}, \frac{3}{2}$ & $(32,5) \frac{1}{2}, \frac{3}{2} \quad(34,1) \frac{1}{2}, \frac{3}{2}$ \\
\hline$(11,2) \frac{1}{2}$ & $(22,1) 1$ & $(33,3) \frac{1}{2}, \frac{3}{2}$ & $(34,1) \frac{1}{2}, \frac{3}{2}$ \\
\hline$(9,3) \frac{1}{2}, \frac{3}{2}$ & $(24,0) 0$ & $(33,3) \frac{1}{2}, \frac{3}{2}$ & \\
\hline$(8,5) \frac{1}{2}, \frac{3}{2}$ & $(24,0) 0$ & $(32,5) \frac{1}{2}, \frac{3}{2}$ & \\
\hline$(10,1) \frac{1}{2}$ & $(24,0) 0$ & $(34,1) \frac{1}{2}$ & \\
\hline$(7,7) \frac{1}{2}$ & $(20,5) 0,1$ & $(27,12) \frac{1}{2}, \frac{3}{2}$ & \\
\hline$(11,2) \frac{1}{2}$ & $(16,10) 0$ & $(27,12) \frac{1}{2}$ & \\
\hline$(4,10) \frac{1}{2}$ & $(16,10) 0$ & $(20,20) \frac{1}{2}$ & \\
\hline
\end{tabular}
of the Hamiltonian (6) contains the so-called 'rotor-like'
TABLE IV: The pseudo SU(3) irreps used in the description of ${ }^{169} \mathrm{Tm}$.

\begin{tabular}{c|ccc} 
Nuclei & $\mathrm{a}$ & $\mathrm{b}$ & $\mathrm{A}_{\text {sym }}$ \\
\hline${ }^{157} \mathrm{Gd}$ & 0.046 & 0.0020 & 0.0008 \\
${ }^{163} \mathrm{Dy}$ & -0.040 & 0.0040 & 0.0016 \\
${ }^{169} \mathrm{Tm}$ & 0.019 & 0.0007 & 0
\end{tabular}

TABLE V: Parameters of the Hamiltonian (6).

terms, used to fine tune the moment of inertia and the position of the different $K$ bands. They have been studied in detail in previous papers where the pseudo $\mathrm{SU}(3)$ symmetry was used as a dynamical symmetry [7]. The strength of these three terms were the only ones adjusted nuclei by nuclei. A detailed analysis of each term of this Hamiltonian and its parametrization has been presented elsewhere 15. In Table $\mathrm{V}$ are shown the current values. These are the same used before for these three nuclei [22, when the proton and neutron subspaces were reduced to $\mathrm{S}=0$ and $\frac{1}{2}$ (in other words, the same set of parameters are working in both approximations of the theory).

The electric quadrupole operator is expressed as 77

$$
Q_{\mu}=e_{\pi} Q_{\pi}+e_{\nu} Q_{\nu} \approx e_{\pi} \frac{\eta_{\pi}+1}{\eta_{\pi}} \tilde{Q}_{\pi}+e_{\nu} \frac{\eta_{\nu}+1}{\eta_{\nu}} \tilde{Q}_{\nu}
$$

with effective charges $e_{\pi}=2.3, e_{\nu}=1.3$. These values are very similar to those used in the pseudo $\mathrm{SU}(3)$ description of even-even nuclei [7, 23]. They are larger than those used in standard calculations of B(E2) strengths 20] due to the passive role assigned to the nucleons in unique parity orbitals, whose contribution to the quadrupole moments is parametrized in this way.

The inclusion of configurations with pseudo-spin 1 and $\frac{3}{2}$ in the Hilbert space allows for a description of several highly excited rotational bands in odd-mass nuclei. Their effect on the M1 transition strengths have been discussed in Ref. 17]. The most relevant result reported there is that, when the configuration space is restricted to states 
with pseudo-spin 0 and $\frac{1}{2}$, it is not possible to find 22] any M1 excitations with sizable strength from the ground state toward the states between 2 to $4 \mathrm{MeV}$ energy region for any of the three nuclei. The M1 strength appears only when the Hilbert space is enlarged to include states with $\tilde{S}_{\pi}$ or $\tilde{S}_{\nu}=1$ or $3 / 2$.

\section{ROTATIONAL BANDS AND SPIN CONTENT}

Fig. 1 shows the yrast and excited normal parity bands in ${ }^{157} \mathrm{Gd}$. Experimental [24 data are plotted on the left hand size of each column, while those obtained using the Hilbert space and the Hamiltonian parameters discussed in the previous sections are shown in the right hand side. The agreement between both is excellent, but for the two higher energy bands the lack of more experimental data in bands $\mathrm{E}$ and $\mathrm{F}$ (see fig. I1) prevents a more rigorous comparison.

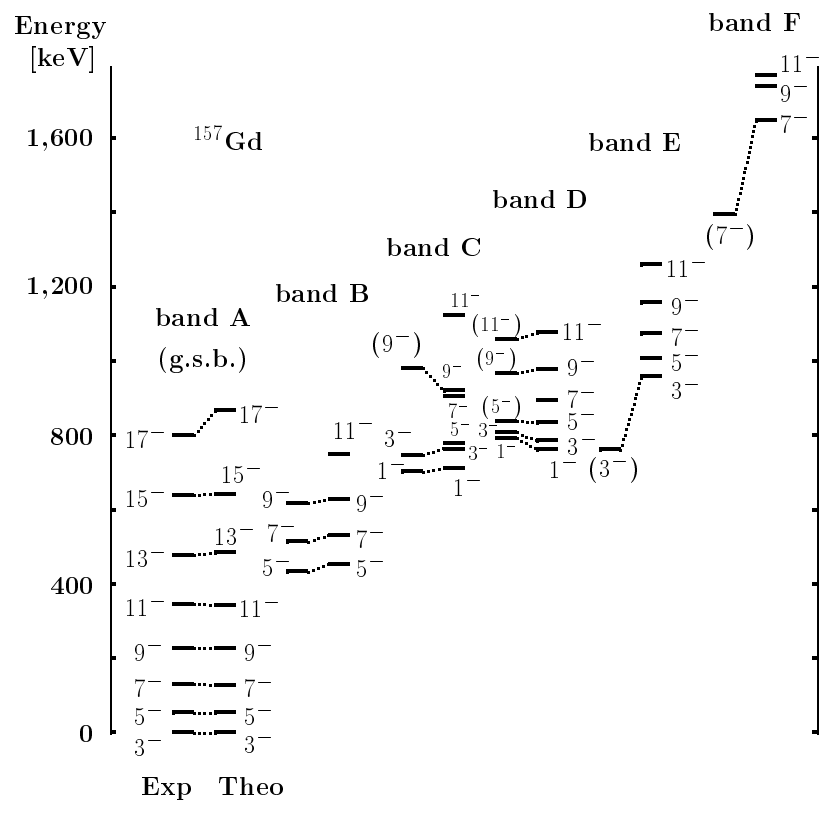

FIG. 1: Negative parity bands in ${ }^{157} \mathrm{Gd}$. The integer numbers indicate twice the total angular momentum of each level.

The whole energy spectra is built up by the interplay between the single-particle and quadrupole-quadrupole terms in the Hamiltonian. The use of realistic singleparticle energies plays a key role in the appropriate ordering of the different band-heads.

Figs. 2 and 3 show the normal parity bands in ${ }^{163}$ Dy and ${ }^{169} \mathrm{Tm}$, respectively. In ${ }^{163} \mathrm{Dy}$ the agreement between theory and experiment is very good for the seven rotational bands (A-G). These seven bands represents nearly all the bands measured. For ${ }^{169} \mathrm{Tm}$ the agreement between the theoretical band structure and its experimental counterpart is still good, although the differ- ences in bandhead energies are larger than in the previous cases.

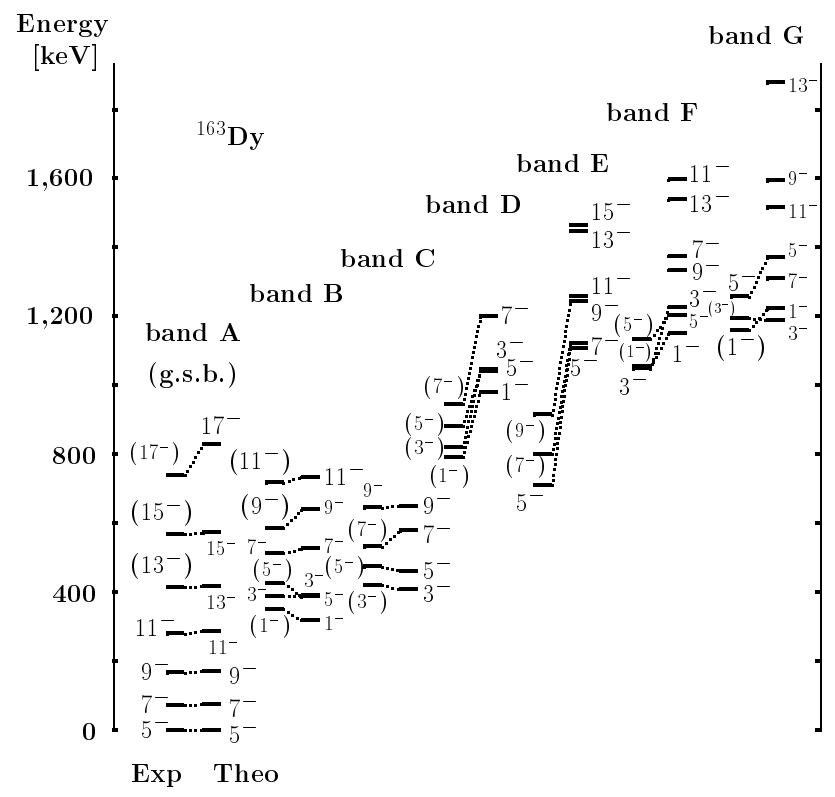

FIG. 2: Negative parity bands in ${ }^{163}$ Dy.

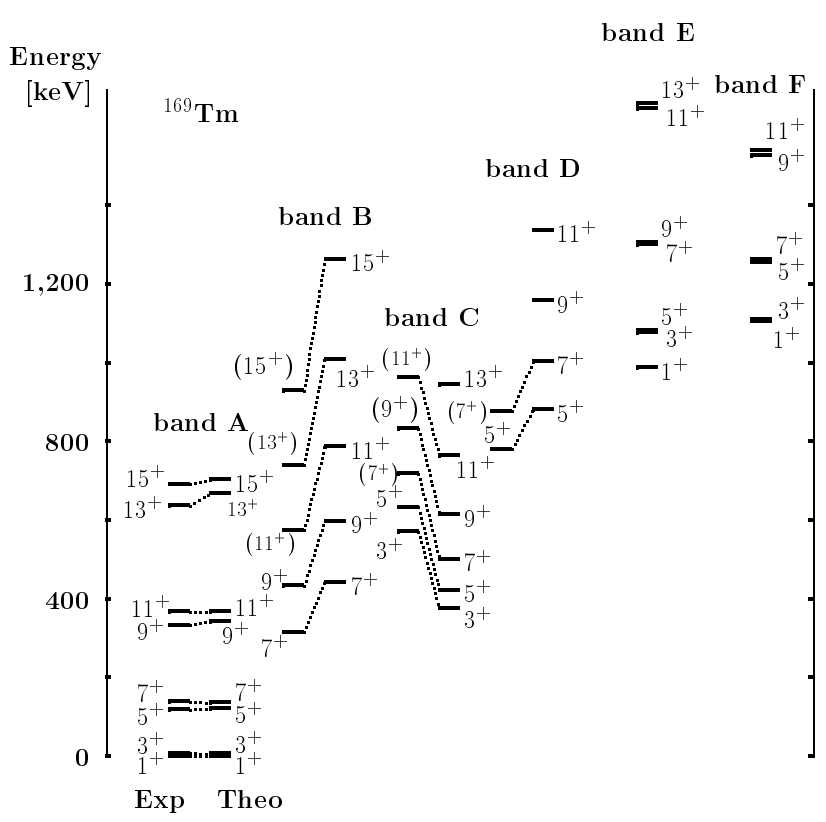

FIG. 3: Positive parity bands in ${ }^{169} \mathrm{Tm}$.

The bands plotted in Figs. (113) have a very regular structure as one moves up the bands. The mixing remains nearly the same for the states with different angular moments belonging to the same band. As a consequence, the pseudo-spin content is practically constant along all the members of each band. Table VI shows the calculated pseudo-spin content of each rotational band in ${ }^{157} \mathrm{Gd},{ }^{163} \mathrm{Dy}$ and ${ }^{169} \mathrm{Tm}$, respectively. As shown in 


\begin{tabular}{cc|cc} 
Nuclei & Band & $\tilde{S}=1 / 2(\%)$ & $\tilde{S}=3 / 2(\%)$ \\
\hline${ }^{157} \mathrm{Gd}$ & $\mathrm{A}$ & 89 & 11 \\
& $\mathrm{~B}$ & 92 & 8 \\
& $\mathrm{C}$ & 92 & 8 \\
& $\mathrm{D}$ & 3 & 97 \\
& $* \mathrm{E}$ & 77 & 23 \\
& $\mathrm{~F}$ & 16 & 84 \\
${ }^{163} \mathrm{Dy}$ & $\mathrm{A}$ & 100 & 0 \\
& $\mathrm{~B}$ & 100 & 0 \\
& $\mathrm{C}$ & 100 & 0 \\
& $\mathrm{D}$ & 100 & 0 \\
& $\mathrm{E}$ & 0 & 100 \\
& $* \mathrm{~F}$ & 100 & 0 \\
& $\mathrm{G}$ & 0 & 100 \\
& & & \\
${ }^{169} \mathrm{Tm}$ & $\mathrm{A}$ & 93 & 7 \\
& $\mathrm{~B}$ & 100 & 0 \\
& $\mathrm{C}$ & 9 & 91 \\
& $\mathrm{D}$ & 100 & 0 \\
& $\mathrm{E}$ & 35 & 65 \\
& $\mathrm{~F}$ & 37 & 63
\end{tabular}

TABLE VI: Pseudo-spin content for each band in ${ }^{157} \mathrm{Gd}$, ${ }^{163} \mathrm{Dy}$ and ${ }^{169} \mathrm{Tm}$. In first column it is indicated the nuclei, the second shows the band, and third and four columns show the $1 / 2$ and $3 / 2$ pseudo spin-content as percentage for each band, respectively.

Eq. (2), each eigenstate is described as a linear combination of pseudo $\mathrm{SU}(3)$ states $|\beta J M\rangle$, with pseudo-spin $\frac{1}{2}$, $\frac{3}{2}$ and $\frac{5}{2}$, depending on the proton and neutron pseudospin couplings.

In most of the bands the mixing is very small, about half of them have no mixing at all. The main contribution of the present work is to add those states with pseudospin $\frac{3}{2}$ (and those marked with $\mathrm{a}^{*}$, see below) to the pseudo $\mathrm{SU}(3)$ description of these nuclei.

The total pseudo-spin content of the nuclear wave function is built with the coupling of the $S_{\pi}$ and $S_{\nu}$ components. In all the cases, the states with pseudo-spin $\tilde{S}=\frac{3}{2}$ are built as $1 \otimes \frac{1}{2}$, and the $0 \otimes \frac{3}{2}$ is not present in the bands analysed. By the other side, although most of the $\tilde{S}=\frac{1}{2}$ come from the coupling $0 \otimes \frac{1}{2}$, the bands marked with a ${ }^{*}$ in Table VI, are built as $\tilde{S}_{\pi}=1 \otimes \tilde{S}_{\nu}=\frac{1}{2}$ for the band $\mathrm{E}$ in ${ }^{157} \mathrm{Gd}$ and $\tilde{S}_{\pi}=\frac{1}{2} \otimes \tilde{S}_{\nu}=1$ for the band $\mathrm{F}$ in $\left.{ }^{163} \mathrm{Dy}\right)$.

\section{B(E2) TRANSITION STRENGTHS}

In Figures 4,5 and 6 are shown the B(E2) intra-band transition strengths for the reported normal parity bands
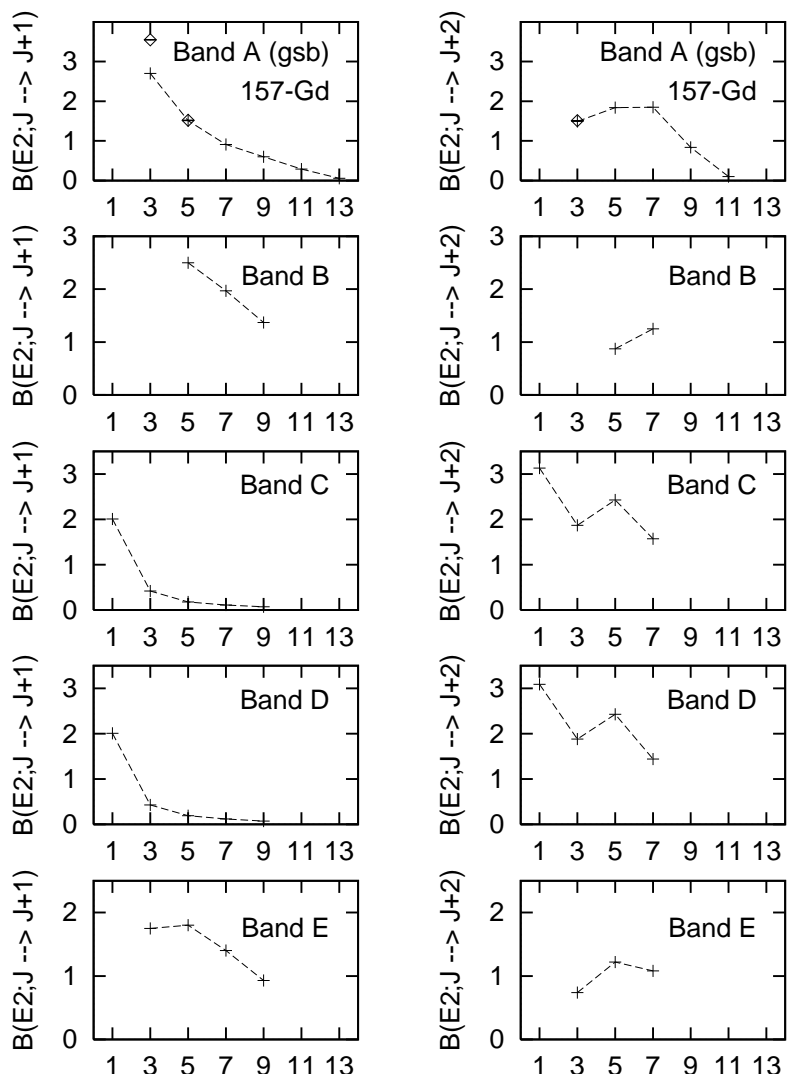

FIG. 4: $\mathrm{B}(\mathrm{E} 2)$ intra-band transitions in ${ }^{157} \mathrm{Gd}\left[e^{2} b^{2}\right]$.

in ${ }^{157} \mathrm{Gd},{ }^{163}$ Dy and ${ }^{169} \mathrm{Tm}$, respectively. All experimental data available [24] have been included with error bars for comparison. For a given band, labeled $\mathrm{A}$ to $\mathrm{G}$, the $\mathrm{x}$ axis represents twice the angular momentum of the initial state for each transition, while the y axis shows the $\mathrm{B}(\mathrm{E} 2)$ strength. The graphs on the left hand side show $\mathrm{B}(\mathrm{E} 2 ; \mathrm{J} \rightarrow \mathrm{J}+1)$ transitions, and those in the right hand side present the $\mathrm{B}(\mathrm{E} 2 ; \mathrm{J} \rightarrow \mathrm{J}+2)$, both in units of $e^{2} b^{2}$. The "+" symbols with segmented lines refers to the calculated values, and the diamonds with error bars to the experimental ones.

In Tables VII, VIII and IX are shown many inter-band $\mathrm{B}(\mathrm{E} 2)$ transition strengths in ${ }^{157} \mathrm{Gd},{ }^{163} \mathrm{Dy}$ and ${ }^{169} \mathrm{Tm}$, respectively. All known experimental data available 24] were included between parenthesis. Theoretical values are in agreement with those reported previously for the lowest energy bands in ${ }^{163}$ Dy [16].

The agreement between the calculated values with those measured is remarkable. Some transitions, like $3 / 2_{C}^{-} \rightarrow 7 / 2_{D}^{-}, 3 / 2_{D}^{-} \rightarrow 7 / 2_{C}^{-}$in ${ }^{157} \mathrm{Gd}$, and $3 / 2_{B}^{-} \rightarrow$ $7 / 2_{C}^{-}, 3 / 2_{D}^{-} \rightarrow 7 / 2_{E}^{-}, 9 / 2_{C}^{-} \rightarrow 11 / 2_{B}^{-}$in ${ }^{163}$ Dy, are larger than $0.05-0.10 e^{2} b^{2}$. It reflects the mixing between the different bands.

The $\mathrm{B}\left(\mathrm{M} 1 ; J_{\text {g.s. }}^{\pi} \rightarrow J_{f}^{\pi}\right)$ transitions in ${ }^{157} \mathrm{Gd},{ }^{163}$ Dy and ${ }^{169} \mathrm{Tm}$ have been presented in Ref. 117. $J_{\text {g.s. }}^{\pi}$ refers to the ground states $\frac{3}{2}^{-}, \frac{5}{2}^{-}$and $\frac{1}{2}^{+}$, respectively, in these 


\begin{tabular}{cccc}
$J i_{\text {band }}^{\pi} \rightarrow J f_{\text {band }}^{\pi} \mathrm{B}(\mathrm{E} 2)$ & $J i_{\text {band }}^{\pi} \rightarrow J f_{\text {band }}^{\pi}$ & $\mathrm{B}(\mathrm{E} 2)$ \\
\hline $1 / 2_{C}^{-} \rightarrow 3 / 2_{A}^{-}$ & 2.6 & $1 / 2_{D}^{-} \rightarrow 3 / 2_{C}^{-}$ & 8.5 \\
$1 / 2_{C}^{-} \rightarrow 5 / 2_{B}^{-}$ & 2.8 & $1 / 2_{D}^{-} \rightarrow 5 / 2_{E}^{-}$ & 2.1 \\
$3 / 2_{C}^{-} \rightarrow 5 / 2_{D}^{-}$ & 3.4 & $3 / 2_{D}^{-} \rightarrow 5 / 2_{C}^{-}$ & 3.1 \\
$3 / 2_{C}^{-} \rightarrow 7 / 2_{D}^{-}$ & 77.1 & $3 / 2_{D}^{-} \rightarrow 7 / 2_{C}^{-}$ & 77.8 \\
$5 / 2_{D}^{-} \rightarrow 7 / 2_{C}^{-}$ & 2.1 & $7 / 2_{A}^{-} \rightarrow 11 / 2_{B}^{-}$ & 2.6 \\
$7 / 2_{B}^{-} \rightarrow 11 / 2_{A}^{-}$ & 2.1 & $7 / 2_{D}^{-} \rightarrow 11 / 2_{A}^{-}$ & 4.3 \\
$7 / 2_{D}^{-} \rightarrow 11 / 2_{B}^{-}$ & 6.6 & $7 / 2_{D}^{-} \rightarrow 11 / 2_{E}^{-}$ & 3.7 \\
$7 / 2_{C}^{-} \rightarrow 11 / 2_{D}^{-}$ & 31.5 & $7 / 2_{E}^{-} \rightarrow 11 / 2_{D}^{-}$ & 14.3 \\
$9 / 2_{B}^{-} \rightarrow 11 / 2_{A}^{-}$ & 3.1 & $9 / 2_{E}^{-} \rightarrow 11 / 2_{D}^{-}$ & 3.7 \\
$9 / 2_{E}^{-} \rightarrow 11 / 2_{C}^{-}$ & 3.3 & &
\end{tabular}

TABLE VII: Calculated B(E2) inter-band transitions in ${ }^{157} \mathrm{Gd}\left[e^{2} b^{2} \times 10^{-2}\right]$.

\begin{tabular}{cccc}
$J i_{\text {band }}^{\pi} \rightarrow J f_{\text {band }}^{\pi}$ & $\mathrm{B}(\mathrm{E} 2)$ & $J i_{\text {band }}^{\pi} \rightarrow J f_{\text {band }}^{\pi}$ & $\mathrm{B}(\mathrm{E} 2)$ \\
\hline $1 / 2_{B}^{-} \rightarrow 3 / 2_{C}^{-}$ & 44.8 & $1 / 2_{D}^{-} \rightarrow 3 / 2_{C}^{-}$ & 2.6 \\
$1 / 2_{F}^{-} \rightarrow 3 / 2_{G}^{-}$ & 14.6 & $1 / 2_{G}^{-} \rightarrow 3 / 2_{D}^{-}$ & 5.6 \\
$1 / 2_{G}^{-} \rightarrow 3 / 2_{F}^{-}$ & 15.6 & $1 / 2_{B}^{-} \rightarrow 5 / 2_{C}^{-}$ & 3.4 \\
$1 / 2_{B}^{-} \rightarrow 5 / 2_{A}^{-}$ & $3.8(4.0 \pm 0.8)$ & $1 / 2_{D}^{-} \rightarrow 5 / 2_{A}^{-}$ & 4.9 \\
$1 / 2_{D}^{-} \rightarrow 5 / 2_{E}^{-}$ & 1.2 & $1 / 2_{F}^{-} \rightarrow 5 / 2_{E}^{-}$ & 4.7 \\
$1 / 2_{F}^{-} \rightarrow 5 / 2_{G}^{-}$ & 1.6 & $1 / 2_{G}^{-} \rightarrow 5 / 2_{E}^{-}$ & 21.7 \\
$3 / 2_{B}^{-} \rightarrow 5 / 2_{A}^{-}$ & $2.3(1.8 \pm 0.6)$ & $3 / 2_{C}^{-} \rightarrow 5 / 2_{B}^{-}$ & 10.7 \\
$3 / 2_{D}^{-} \rightarrow 5 / 2_{A}^{-}$ & 1.6 & $3 / 2_{D}^{-} \rightarrow 5 / 2_{C}^{-}$ & 2.1 \\
$3 / 2_{D}^{-} \rightarrow 5 / 2_{E}^{-}$ & 32.1 & $3 / 2_{G}^{-} \rightarrow 5 / 2_{E}^{-}$ & 13.7 \\
$3 / 2_{G}^{-} \rightarrow 5 / 2_{F}^{-}$ & 2.8 & $3 / 2_{F}^{-} \rightarrow 5 / 2_{G}^{-}$ & 1.9 \\
$3 / 2_{B}^{-} \rightarrow 7 / 2_{A}^{-}$ & $0.3(3.7 \pm 1.6)$ & $3 / 2_{B}^{-} \rightarrow 7 / 2_{C}^{-}$ & 97.9 \\
$3 / 2_{C}^{-} \rightarrow 7 / 2_{A}^{-}$ & 3.0 & $3 / 2_{C}^{-} \rightarrow 7 / 2_{B}^{-}$ & 28.4 \\
$3 / 2_{D}^{-} \rightarrow 7 / 2_{A}^{-}$ & 1.9 & $3 / 2_{D}^{-} \rightarrow 7 / 2_{B}^{-}$ & 1.2 \\
$3 / 2_{D}^{-} \rightarrow 7 / 2_{E}^{-}$ & 62.2 & $3 / 2_{D}^{-} \rightarrow 7 / 2_{G}^{-}$ & 1.9 \\
$3 / 2_{G}^{-} \rightarrow 7 / 2_{E}^{-}$ & 45.3 & $3 / 2_{G}^{-} \rightarrow 7 / 2_{F}^{-}$ & 5.3 \\
$3 / 2_{F}^{-} \rightarrow 7 / 2_{G}^{-}$ & 5.1 & $5 / 2_{B}^{-} \rightarrow 5 / 2_{A}^{-}$ & 0.4 \\
$5 / 2_{B}^{-} \rightarrow 7 / 2_{A}^{-}$ & $2.0(3.7 \pm 2.1)$ & $5 / 2_{B}^{-} \rightarrow 7 / 2_{C}^{-}$ & 11.5 \\
$5 / 2_{E}^{-} \rightarrow 7 / 2_{D}^{-}$ & 6.3 & $5 / 2_{E}^{-} \rightarrow 7 / 2_{G}^{-}$ & 45.4 \\
$5 / 2_{B}^{-} \rightarrow 9 / 2_{A}^{-}$ & $2.2(3.0 \pm 1.6)$ & $5 / 2_{C}^{-} \rightarrow 9 / 2_{A}^{-}$ & 1.7 \\
$5 / 2_{D}^{-} \rightarrow 9 / 2_{A}^{-}$ & 2.2 & $5 / 2_{G}^{-} \rightarrow 9 / 2_{E}^{-}$ & 3.2 \\
$7 / 2_{C}^{-} \rightarrow 9 / 2_{B}^{-}$ & 11.4 & $7 / 2_{E}^{-} \rightarrow 9 / 2_{D}^{-}$ & 1.5 \\
$7 / 2_{E}^{-} \rightarrow 9 / 2_{F}^{-}$ & 1.2 & $7 / 2_{C}^{-} \rightarrow 11 / 2_{A}^{-}$ & 2.2 \\
$7 / 2_{E}^{-} \rightarrow 11 / 2_{G}^{-}$ & 2.0 & $7 / 2_{G}^{-} \rightarrow 11 / 2_{E}^{-}$ & 8.1 \\
$7 / 2_{F}^{-} \rightarrow 11 / 2_{G}^{-}$ & 1.3 & $9 / 2_{B}^{-} \rightarrow 11 / 2_{C}^{-}$ & 4.7 \\
$9 / 2_{C}^{-} \rightarrow 11 / 2_{B}^{-}$ & 63.8 & $9 / 2_{E}^{-} \rightarrow 11 / 2_{G}^{-}$ & 22.5 \\
$9 / 2_{B}^{-} \rightarrow 13 / 2_{A}^{-}$ & 3.2 & $9 / 2_{C}^{-} \rightarrow 13 / 2_{A}^{-}$ & 1.3 \\
$9 / 2_{D}^{-} \rightarrow 13 / 2_{A}^{-}$ & 1.9 & $11 / 2_{B}^{-} \rightarrow 13 / 2_{C}^{-}$ & 33.8 \\
$11 / 2_{C}^{-} \rightarrow 13 / 2_{A}^{-}$ & 1.0 & $11 / 2_{C}^{-} \rightarrow 13 / 2_{B}^{-}$ & 7.5 \\
$11 / 2_{C}^{-} \rightarrow 15 / 2_{A}^{-}$ & 1.7 & &
\end{tabular}

TABLE VIII: B(E2) inter-band transitions in ${ }^{163}$ Dy. The experimental data are given between parenthesis $\left[e^{2} b^{2} \times 10^{-2}\right]$.
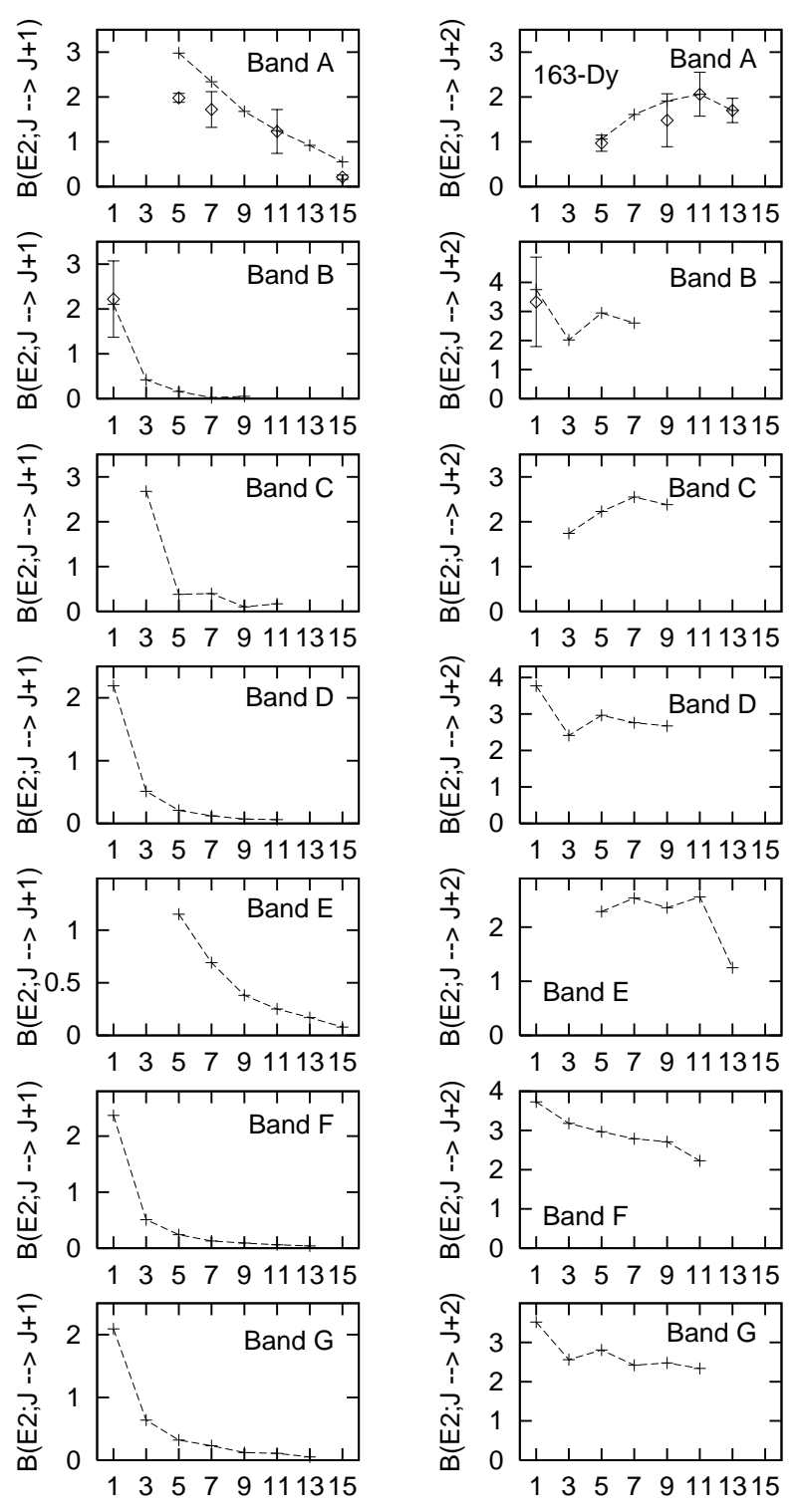

FIG. 5: B(E2) intra-band transitions in ${ }^{163}$ Dy $\left[e^{2} b^{2}\right]$.

nuclei. In that work it was shown that most of the states with energies between 2 and $4 \mathrm{MeV}$ have very important contributions from states with proton and neutron pseudo-spin 1 and $\frac{3}{2}$.

\section{SUMMARY AND CONCLUSIONS}

The pseudo SU(3) shell model for odd-mass nuclei has been shown to offer a quantitative microscopic description of several normal parity rotational bands in ${ }^{157} \mathrm{Gd}$, ${ }^{163} \mathrm{Dy}$ and ${ }^{169} \mathrm{Tm}$. The present article complements the study of the scissors mode in these nuclei, their fragmentation and their summed $\mathrm{B}(\mathrm{M} 1 ; \uparrow)$ strengths.

In order to successfully describe the excited bands, 

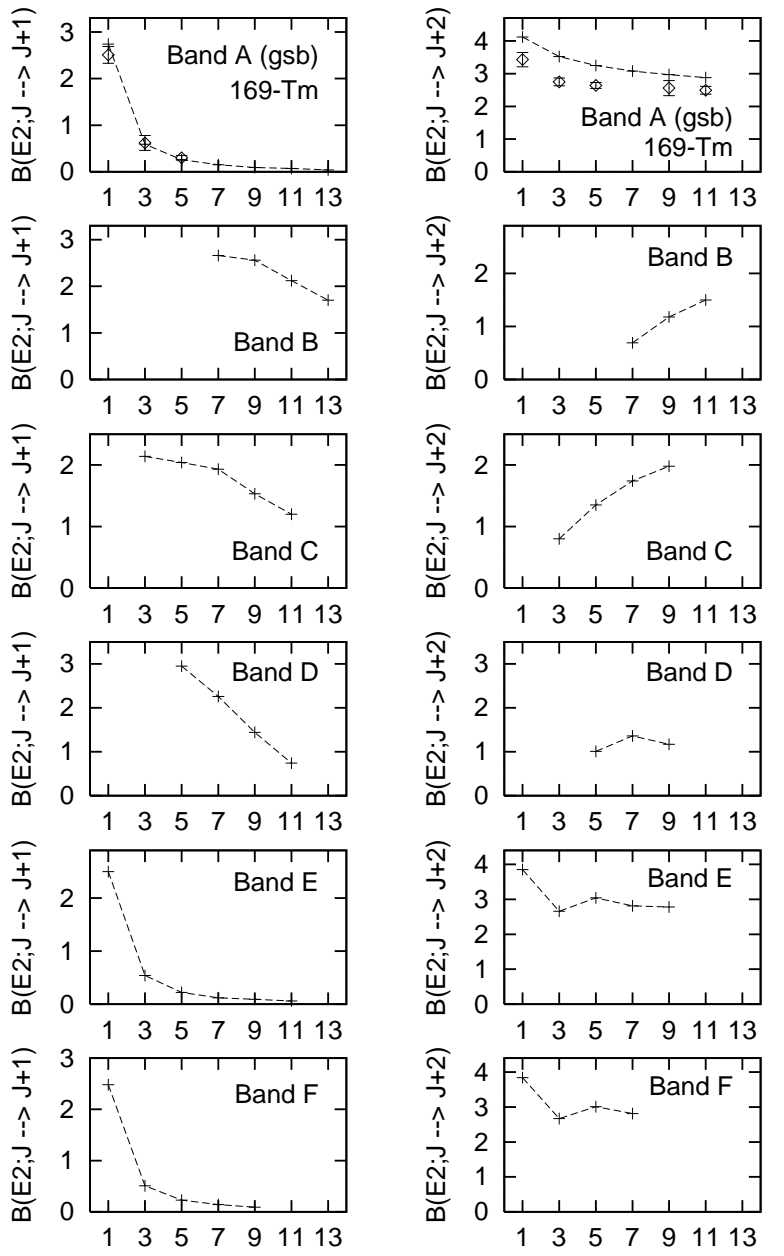

FIG. 6: B(E2) intra-band transitions in ${ }^{169} \mathrm{Tm}\left[e^{2} b^{2}\right]$.

intra- and inter-band $\mathrm{B}(\mathrm{E} 2)$ and $\mathrm{B}(\mathrm{M} 1)$ transition strengths, it was necessary to enlarge the Hilbert space, including those pseudo $\mathrm{SU}(3)$ irreps with the largest $C_{2}$ values having pseudo-spin 1 and $\frac{3}{2}$ and to use realistic values for the single-particle energies. This expansion of the model space allowed for the description of new low lying normal parity bands, in agreement with the experimental data. The new bands have predominantly $\tilde{S}=\frac{3}{2}$, but it was shown that the most important contribution comes from the proton or neutron subspaces with $\tilde{S}=1$. It implies that the pseudo-spin mixing in the wave function takes place mostly in the sub-space with even number of particles. The interplay between the single-particle and the quadrupole-quadrupole terms in the Hamiltonian defines this mixing. The delicated balance between these two interactions, defines the gross features of the calculated excited bands.

Intra- and inter-band $\mathrm{B}(\mathrm{E} 2)$ transition strengths are predicted in good agreement with their measured values and with those reported previously with the pseudo $\mathrm{SU}(3)$ model. Detailed description of magnetic proper-

\begin{tabular}{cc}
$J i_{\text {band }}^{\pi} \rightarrow J f_{\text {band }}^{\pi}$ & $\mathrm{B}(\mathrm{E} 2)$ \\
\hline $1 / 2_{E}^{+} \rightarrow 3 / 2_{C}^{+}$ & 1.2 \\
$1 / 2_{E}^{+} \rightarrow 3 / 2_{F}^{+}$ & 7.9 \\
$1 / 2_{F}^{+} \rightarrow 3 / 2_{C}^{+}$ & 1.1 \\
$1 / 2_{F}^{+} \rightarrow 3 / 2_{E}^{+}$ & 9.0 \\
$1 / 2_{E}^{+} \rightarrow 5 / 2_{C}^{+}$ & 1.0 \\
$3 / 2_{A}^{+} \rightarrow 5 / 2_{C}^{+}$ & 0.004 \\
$3 / 2_{C}^{+} \rightarrow 5 / 2_{A}^{+}$ & 0.107 \\
$3 / 2_{E}^{+} \rightarrow 5 / 2_{C}^{+}$ & 0.011 \\
$3 / 2_{E}^{+} \rightarrow 5 / 2_{D}^{+}$ & 0.001 \\
$3 / 2_{E}^{+} \rightarrow 5 / 2_{F}^{+}$ & 3.5 \\
$3 / 2_{F}^{+} \rightarrow 5 / 2_{C}^{+}$ & 1.5 \\
$3 / 2_{F}^{+} \rightarrow 5 / 2_{E}^{+}$ & 1.7 \\
$3 / 2_{A}^{+} \rightarrow 7 / 2_{B}^{+}$ & $0.00168(0.0006 \pm 0.0001)$ \\
$3 / 2_{A}^{+} \rightarrow 7 / 2_{C}^{+}$ & 0.012 \\
$3 / 2_{A}^{+} \rightarrow 7 / 2_{D}^{+}$ & 0.029 \\
$3 / 2_{C}^{+} \rightarrow 7 / 2_{A}^{+}$ & 0.015 \\
$3 / 2_{C}^{+} \rightarrow 7 / 2_{D}^{+}$ & 0.005 \\
$3 / 2_{F}^{+} \rightarrow 7 / 2_{C}^{+}$ & 0.9 \\
$5 / 2_{D}^{+} \rightarrow 7 / 2_{B}^{+}$ & 1.1 \\
$5 / 2_{A}^{+} \rightarrow 7 / 2_{B}^{+}$ & $0.0037(0.0014 \pm 0.0002)$ \\
$5 / 2_{E}^{+} \rightarrow 7 / 2_{C}^{+}$ & 1.1 \\
$5 / 2_{E}^{+} \rightarrow 7 / 2_{F}^{+}$ & 2.4 \\
$5 / 2_{F}^{+} \rightarrow 7 / 2_{E}^{+}$ & 23.6 \\
$5 / 2_{D}^{+} \rightarrow 9 / 2_{B}^{+}$ & 0.9 \\
$5 / 2_{E}^{+} \rightarrow 9 / 2_{C}^{+}$ & 1.1 \\
$7 / 2_{A}^{+} \rightarrow 7 / 2_{B}^{+}$ & $0.00611(0.00176 \pm 0.00005)$ \\
&
\end{tabular}

TABLE IX: B(E2) inter-band transitions in ${ }^{169} \mathrm{Tm}$. Experimental data available are between parenthesis $\left[e^{2} b^{2} \times 10^{-2}\right]$

ties of these rotational bands [25] and the excited bands in other rare-earth nuclei are desirable.

\section{ACKNOWLEDGEMENTS}

This work was supported in part by CONACyT (México) and the US National Science Foundation.
[1] M. G. Mayer, Phys. Rev. 75, 1969 (1949); O. Haxel, J. H. D. Jenssen, and H. E. Suess, Phys. Rev. 75, 1766 (1949).

[2] B. A. Brown and B .H. Wildenthal, Ann. Rev. Nucl. Part.
Sc. 38, 29 (1988).

[3] E. Caurier, et. al. Phys. Rev. Lett. 75, 2466 (1995); A. Schmidt, et. al. Phys. Rev. C 62, 044319 (2000). 
[4] M. Valliéres and $\mathrm{H}$. Wu, in Computational Nuclear Physics 1 edited by K. Langanke, J. A. Maruhn, and S. E. Koonin (Springer, Berlin, 1991).

[5] J. P. Elliott, Proc. Roy. Soc. London Ser. A 245, 128 (1958); 245, 562 (1958).

[6] K. T. Hecht and A. Adler, Nucl. Phys. A 137, 129 (1969); A. Arima, M. Harvey, and K. Shimizu, Phys. Lett. B 30, 517 (1969).

[7] J. P. Draayer, et. al., Nucl. Phys. A 381, 1 (1982); J. P. Draayer and K. J. Weeks, Ann. of Phys. 156, 41 (1984); O. Castaños, et. al., Ann. of Phys. 329, 290 (1987).

[8] A. L. Blokhin, et. al., Phys. Rev. Lett. 74, 4149 (1995); J. N. Ginocchio, Phys. Rev. Lett. 78, 436 (1997); J. Meng, et. al. Phys. Rev. C 58, R632 (1998).

[9] C. Bahri and J.P. Draayer, Comput. Phys. Commun. 83, 59 (1994).

[10] T. Beuschel, J.G. Hirsch, and J.P. Draayer, Phys. Rev. C 61, 54307 (2000).

[11] G. Popa, J. G. Hirsch and J. P. Draayer, Phys. Rev. C 62, 064313 (2000).

[12] J. P. Draayer, G. Popa and J. G. Hirsch, Acta Phys. Pol. B 32, 2697 (2001).

[13] C. Vargas, J. G. Hirsch, T. Beuschel, J. P. Draayer, Phys. Rev. C 61, 31301 (2000).

[14] J. G. Hirsch, C.E. Vargas, and J. P. Draayer, Rev. Mex.
Fis. 46 Supl. 1, 54 (2000).

[15] C.E. Vargas, J. G. Hirsch and J.P. Draayer, Nucl. Phys. A 673, 219-237 (2000).

[16] C. Vargas, J. G. Hirsch, J. P. Draayer, Phys. Rev. C 64, 034306 (2001).

[17] C. E. Vargas, J. G. Hirsch, J. P. Draayer, Subbmited to Phys. Rev. Lett.

[18] C. E. Vargas, J. G. Hirsch, J. P. Draayer, Nucl. Phys. A 690, 409 (2001); ibid, Nucl. Phys. A 697, 655 (2002)

[19] C. Vargas, J. G. Hirsch, P. O. Hess, and J. P. Draayer, Phys. Rev. C 58, 1488 (1998); J. P. Draayer, and K. J. Weeks, Ann. Phys. 156, 41 (1984).

[20] P. Ring and P. Schuck. The Nuclear Many-Body Problem, Springer, Berlin (1979).

[21] M. Dufour and A. P. Zuker, Phys. Rev. C 54, 1641 (1996).

[22] PhD Thesis, Carlos E. Vargas, CINVESTAV 2001, México.

[23] T. Beuschel, J.P. Draayer, D. Rompf , and J.G. Hirsch, Phys. Rev. C 57, 1233 (1998).

[24] National Nuclear Data Center, http://bnlnd2.dne.bnl.gov

[25] A. Stuchbery, Nucl. Phys. A 700, 83 (2002). 